\title{
Three-Dimensional Perovskite Methylhydrazinium Lead Chloride with Two Polar Phases and Unusual Second-Harmonic Generation Bistability above Room Temperature
}

Mirosław Mączka,* Anna Gagor, Jan K. Zarȩba, Dagmara Stefanska, Marek Drozd, Sergejus Balciunas, Mantas Simenas, Juras Banys, and Adam Sieradzki

Cite This: Chem. Mater. 2020, 32, 4072-4082

Read Online

ABSTRACT: Formation of noncentrosymmetric three-dimensional (3D) lead halide perovskites has been a widely sought after goal because the polar structure opens up new vistas to properties of these materials, e.g., improved charge separation for photovoltaics arising from ferroelectric order. Here, we report growth and unique properties of a new highly distorted $3 \mathrm{D}$ perovskite, methylhydrazinium lead chloride $\left(\mathrm{CH}_{3} \mathrm{NH}_{2} \mathrm{NH}_{2} \mathrm{PbCl}_{3}, \mathbf{M H y P b C l}_{3}\right)$. This perovskite crystallizes in polar $\mathrm{P2}_{1}$ structure at room temperature, which consists of two types of $\mathrm{PbCl}_{6}$ octahedra: one weakly and another strongly deformed. The unusual deformation of every second perovskite layer is forced by the large size of methylhydrazinium cations and the ability of $\mathrm{NH}_{2}{ }^{+}$terminal groups of

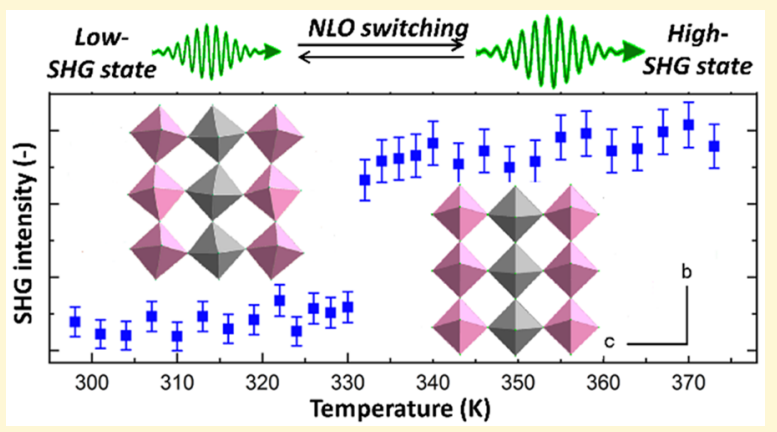
methylhydrazinium cations to form coordination bonds with $\mathrm{Pb}^{2+}$ metal centers. On heating, $\mathbf{M H y P b C l}_{3}$ undergoes a phase transition at $342 \mathrm{~K}$ into another polar $\mathrm{Pb} 2_{1} m$ phase with ordered organic cations. Temperature-resolved second-harmonic generation (TR-SHG) measurements confirm acentricity of both phases and show that second-harmonic response is enhanced for the high-temperature $\mathrm{Pb}_{1} \mathrm{~m}$ phase. This intriguing property of $\mathbf{M H y P b C l}_{3}$ has been employed to demonstrate an unprecedented kind of quadratic nonlinear optical switching in which a second-harmonic response is switched between a room-temperature, low-SHG state and a high-temperature, high-SHG state. X-ray diffraction shows that enhancement of polar properties is due to rearrangement of the perovskite's organic substructure. There is a clear pyrocurrent peak, but switching of the electric polarization could not be observed. Optical studies showed that $\mathbf{M H y P b C l}_{3}$ is a wide-bandgap material with a bandgap of $3.4 \mathrm{eV}(365 \mathrm{~nm})$. At low temperatures, it exhibits weak UV emissions at 362 and $369 \mathrm{~nm}$ as well as a strong broadband white emission.

\section{INTRODUCTION}

Hybrid organic-inorganic compounds have been the subject of intense studies because their diverse structural and chemical variability offers unlimited opportunities for tuning their physical and chemical properties by chemical modification of the organic and/or inorganic parts. One of the most important subgroups of hybrid perovskites is metal halides, especially three-dimensional (3D) perovskites with formula $\mathrm{ABX}_{3}$, where $\mathrm{A}$ is an organic or alkali metal cation, $\mathrm{B}$ is a divalent cation (typically $\mathrm{Pb}^{2+}, \mathrm{Sn}^{2+}$, or $\mathrm{Ge}^{2+}$ ), and $\mathrm{X}$ stands for halogen. ${ }^{1-5} 3 \mathrm{D}$ halide perovskites, especially the iodide subfamily, have enjoyed tremendous attention in recent years because of their unique properties that raised high hopes regarding applications in solar cells. ${ }^{2,4,6}$ While $3 \mathrm{D}$ lead halides are promising photovoltaic materials, they also present many other interesting properties such as photoluminescence (PL), lasing, and scintillating properties. $^{1-3,5,7,8}$ Lead chlorides attracted less attention than bromides and iodides because their large bandgaps $(>2.8 \mathrm{eV})$ make them unsuitable for single-junction solar-cell applications.
They are, however, suitable candidates for UV light-emitting devices and photodetectors. ${ }^{9,10}$

Excellent optoelectronic properties of 3D halides promoted broad interest in the search for new 3D analogues. Unfortunately, the structural tunability of $3 \mathrm{D}$ lead halides is highly limited because cavities formed in the lead halide frameworks may accommodate only the smallest organic cations. Thus, the $3 \mathrm{D}$ structure is predicted to be stable when the tolerance factor is between 0.8 and $1.0,{ }^{1}$ and this condition is satisfied only for cesium, methylammonium $\left(\mathrm{MA}^{+}\right)$, hydroxylammonium $\left(\mathrm{NH}_{3} \mathrm{OH}^{+}\right)$, hydrazinium $\left(\mathrm{NH}_{2} \mathrm{NH}_{3}^{+}\right)$, azetidinium $\left(\left(\mathrm{CH}_{2}\right)_{3} \mathrm{NH}_{2}^{+}\right)$, formamidinium $\left(\mathrm{FA}^{+}\right)$, and imidazolium

Received: March 5, 2020

Revised: April 12, 2020

Published: April 13, 2020 
$\left(\mathrm{C}_{3} \mathrm{~N}_{2} \mathrm{H}_{5}^{+}\right)$cations. ${ }^{1}$ Experimental studies show that the real situation is even worse because $3 \mathrm{D}$ perovskites containing corner-sharing $\mathrm{PbX}_{6}$ octahedra thus far comprised only $\mathrm{MA}^{+}$, $\mathrm{FA}^{+}$, or $\mathrm{Cs}^{+}$as cations, while for the remaining small organic cations, a nonperovskite or layered perovskite structure were formed. ${ }^{1-14}$ It is worth adding that, most recently, a new 3D lead halide perovskite-related ferroelectric [2trimethylammonioethylammonium $] \mathrm{Pb}_{2} \mathrm{Cl}_{6}$ was reported that, however, has a different lead chloride framework of both cornerand edge-sharing $\mathrm{PbCl}_{6}$ octahedra. ${ }^{15}$

It is well-known that the highest-temperature phases of $\mathrm{MAPbX}_{3}, \mathrm{FAPbX}_{3}$, and $\mathrm{CsPbX}$ have cubic symmetry (space group $P m \overline{3} m$ ), but they undergo successive phase transitions on cooling to tetragonal and then orthorhombic or trigonal phases, which according to diffraction studies are centrosymmetric. ${ }^{2,16-20}$ Very recently, we have employed methylhydrazinium cation $\left(\mathrm{CH}_{3} \mathrm{NH}_{2} \mathrm{NH}_{2}^{+}, \mathrm{MHy}^{+}\right)$in the construction of organicinorganic hybrid compounds ${ }^{21-23}$ and discovered that the lead halide framework may also accommodate $\mathrm{MHy}^{+}$in spite of the fact that this cation has a significantly larger ionic radius $(264$ $\mathrm{pm})$ than $\mathrm{FA}^{+}(253 \mathrm{pm}){ }^{1,21}$ This is evident in the formation of $\mathrm{MHyPbBr}_{3}$ 3D perovskite, for which the tolerance factor is predicted to be $1.03 .^{23}$ The high-temperature (HT) phase of $\mathrm{MHyPbBr}_{3}$ has the same cubic symmetry (space group $P m \overline{3} \mathrm{~m}$ ) as the highest-temperature phases of $\mathrm{MAPbX}_{3}, \mathrm{FAPbX}_{3}$, and $\mathrm{CsPbX}$. However, unlike archetypal $\mathrm{MAPbX}_{3}, \mathrm{FAPbX}_{3}$, and $\mathrm{CsPbX}_{3}$ analogues, the room-temperature (RT) structure of $\mathbf{M H y P b B r}_{3}$ shows exceptionally large framework distortion and is strongly noncentrosymmetric, as evidenced by secondharmonic generation (SHG) studies. ${ }^{23}$ We have also showed that $\mathbf{M H y P b B r}_{3}$ features a switchable dielectric behavior, associated with phase transition at $418 \mathrm{~K}$ into the $P m \overline{3} m$ phase as well as thermochromism and two-photon energy upconversion under $800 \mathrm{~nm}$ excitation. ${ }^{23}$

Here, we report the synthesis of a second 3D perovskite comprising $\mathrm{MHy}^{+}$cation, i.e., $\mathbf{M H y P b C l}_{3}$. This $3 \mathrm{D}$ perovskite is an analogue to $\mathbf{M H y P b B r}_{3}$, but its properties related to polar order are even richer because, on either side of the above-roomtemperature phase transition, this material exhibits noncentrosymmetric structure. Quite intriguingly, the high-temperature (HT) phase was found to be more polar than the RT phase. The unusual enhancement of the SHG response that occurs upon transition to the HT phase was employed to demonstrate an unprecedented type of quadratic nonlinear optical (NLO) switching in which the SHG response is switched between RT low-SHG state and HT high-SHG state. Insights on the optical and electrical properties as well as the phasetransition mechanism are provided, unequivocally showing that $\mathbf{M H y P b C l}_{3}$ reveals disparate properties compared to the $\mathbf{M H y P b B r}$ analogue, as well as different properties than those of $\mathrm{FAPbCl}_{3}, \mathrm{MAPbCl}_{3}$, and $\mathrm{CsPbCl}_{3}$ chloride-based analogues.

\section{EXPERIMENTAL SECTION}

Synthesis. $\mathrm{PbCl}_{2}$ (98\%, Sigma-Aldrich), methylhydrazine (98\%, Sigma-Aldrich), hydrochloric acid (37 wt \% in $\mathrm{H}_{2} \mathrm{O}$, Sigma-Aldrich), $\mathrm{N}, \mathrm{N}$-dimethylformamide (DMF, $99.8 \%$ ), and methyl acetate $(99.5 \%$, Sigma-Aldrich) were commercially available and used without further purification. To grow single crystals, $\mathrm{HCl}$ was added dropwise to 2 mmol of methylhydrazine until $\mathrm{pH}=7$. Then $3 \mathrm{~mL}$ of DMF and $2 \mathrm{mmol}$ of $\mathrm{PbCl}_{2}$ was added under continuous stirring, and the mixture was heated to $40^{\circ} \mathrm{C}$ and stirred for $1 \mathrm{~h}$. Because after this time not all $\mathrm{PbCl}_{2}$ was dissolved, a small amount of DMF was added until complete dissolution of $\mathrm{PbCl}_{2}$. The solution was placed in a glass vial, and this vial was placed in a second larger glass vial containing methyl acetate. The lid of the outer vial was thoroughly sealed, whereas the lid of the inner vial was loosened to allow diffusion of methyl acetate into the precursor solution. Colorless transparent crystals with dimensions up to $1.5 \mathrm{~mm}$ were harvested after 5 days, filtered from the mother liquid, and dried at room temperature. A good match of their powder X-ray diffrction (XRD) patterns with the calculated ones based on the single-crystal data (Figure S1 in the Supporting Information) confirmed the phase purity of the bulk sample.

Caution! Methylhydrazine is toxic and must be handled with extreme caution and the appropriate protective gear.

DSC and Thermogravimetric Measurements. Heat capacity was measured using a Mettler Toledo DSC-1 calorimeter with a high resolution of $0.4 \mu \mathrm{W}$. Nitrogen was used as a purging gas, and the heating and cooling rates were $5 \mathrm{~K} / \mathrm{min}$. The sample weight was 29.01 $\mathrm{mg}$. The excess heat capacity associated with the phase transitions was evaluated by subtraction from the data the baseline representing variation in the absence of the phase transitions. A thermogravimetric analysis (TGA) study was performed in the temperature range 300$1123 \mathrm{~K}$ using a PerkinElmer TGA 4000. The sample weight was ca. $17.56 \mathrm{mg}$, and the heating speed rate was $10 \mathrm{~K} / \mathrm{min}$. Pure nitrogen gas as an atmosphere was used.

X-ray Powder Diffraction. A powder XRD pattern was obtained on an X'Pert PRO X-ray diffraction system equipped with a PIXcel ultrafast line detector and Soller slits for $\mathrm{Cu} K \alpha_{1}$ radiation $(\lambda=1.54056$ $\AA$ ). The powder was measured in the reflection mode, and the X-ray tube settings were $30 \mathrm{~mA}$ and $40 \mathrm{kV}$.

SHG. SHG studies were performed using a laser system consisting of a Quantronix Integra-C regenerative amplifier operating as an $800 \mathrm{~nm}$ pump and a Quantronix-Palitra-FS BIBO crystal-based optical parametric amplifier (OPA). This system delivers wavelength-tunable pulses of $\sim 130$ fs length and operates at a repetition rate of $1 \mathrm{kHz}$. In this study we have used the output from the Quantronix-Palitra-FS femtosecond OPA set to $1300 \mathrm{~nm}$ wavelength.

Assessment of SHG efficiency of $\mathbf{M H y P b C l}{ }_{3}$ was performed with the use of the Kurtz-Perry technique. Potassium dihydrogen phosphate (KDP) was used as a SHG reference. The single crystals of $\mathbf{M H y P b C l}_{3}$ and KDP were crushed with a spatula and sieved through a mini-sieve set (Aldrich), allowing for collection of a microcrystal size fraction of 125-177 $\mu \mathrm{m}$. Next, size-graded samples of $\mathbf{M H y P b C l}_{3}$ and $\mathrm{KDP}$ were fixed between microscope glass slides (forming tightly packed layers), sealed, and mounted to the sample holder. The Kurtz-Perry test was performed at $298 \mathrm{~K}$ for $1300 \mathrm{~nm}$ wavelength, at an average beam power of $120 \mathrm{~mW}$. Spectra were collected for 750 and $1000 \mathrm{~ms}$ for KDP and $\mathrm{MHyPbCl}_{3}$, respectively (averaged 5 times).

The laser beam was directed onto samples at $45^{\circ}$ and was unfocused in all cases. Signal-collecting optics, mounted to the glass optical fiber, was placed perpendicularly to the plane of the sample (backscattering geometry). Scattered pumping radiation was suppressed with the use of a $700 \mathrm{~nm}$ short-pass dielectric filter (FESH0700, Thorlabs). All emission spectra were recorded by an Ocean Optics Flame spectrograph.

The examination of the SHG response in the function of the temperature phase was conducted in a separate measurement (using a $1300 \mathrm{~nm}$ beam, in which the sample was mounted to a thermostated heating plate (Heidolph) equipped with an external thermocouple). The sample-preparation protocol was the same as for the Kurtz-Perry test. For TR-SHG the average power used was $99 \mathrm{~mW}$; for the switching study the beam was attenuated to $49 \mathrm{~mW}$. Spectra were collected for $2500 \mathrm{~ms}$ at each temperature point and averaged two times.

Caution! Working with the high-power laser is dangerous to the eyes, especially in the spectral range in which the beam is invisible. Adequate eye protection should be used during measurements.

Dielectric Properties. For dielectric and pyrocurrent measurements, we used a cylindrical pellet sample $(5 \mathrm{~mm}$ in diameter and 1.68 $\mathrm{mm}$ in thickness) pressed from $\mathrm{MHyPbCl}_{3}$ powder using a uniaxial press (pressure $<200 \mathrm{MPa}$ ). Electric field-dependent polarization measurements were performed on a single-crystal sample of size $0.8 \times 1$ $\times 1 \mathrm{~mm}^{3}$. In both cases, electrodes were deposited using a silver paste.

Dielectric measurements were performed in the $100 \mathrm{~Hz}-1 \mathrm{MHz}$ frequency range using an HP 4284A LCR meter. A parallel-plate 

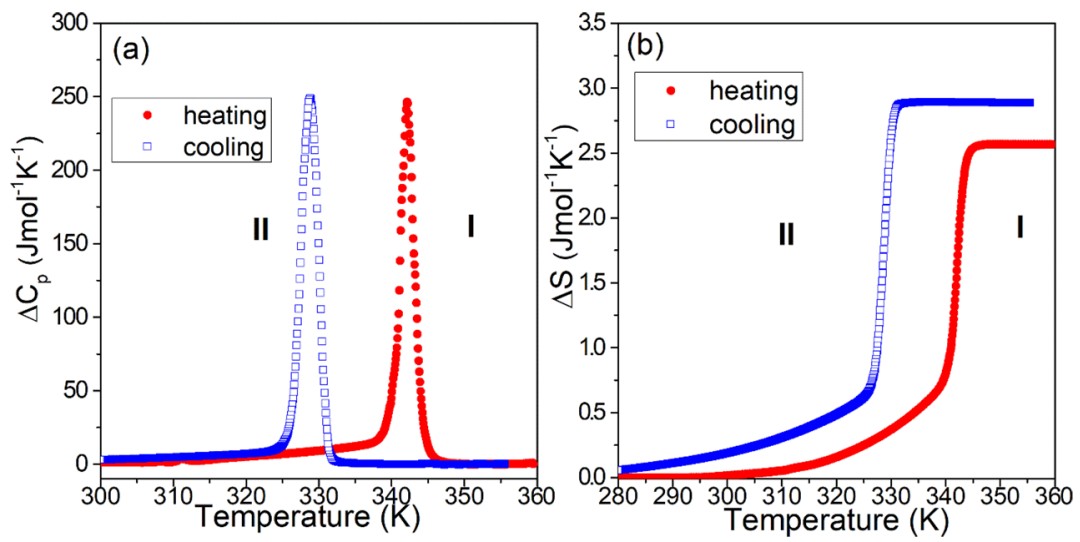

Figure 1. Change in (a) $C_{p}$ and (b) $S$ related to the phase transition in the heating (red) and cooling (blue) runs.

capacitor model was used to calculate the complex dielectric permittivity. Experiments were performed during cooling at a rate of $1 \mathrm{~K} / \mathrm{min}$.

Prior to pyrocurrent measurement, the pellet sample was heated to $355 \mathrm{~K}$ and then cooled down to $150 \mathrm{~K}$. During cooling, an electric poling field of $180 \mathrm{~V} / \mathrm{cm}$ was applied. At $150 \mathrm{~K}$, the sample electrodes were shorted for 15 min using a $10 \mathrm{k} \Omega$ resistor. Current measurements were performed using a Keithley 6514 electrometer during heating of the sample from 190 to $347 \mathrm{~K}$ with a heating rate of $3 \mathrm{~K} / \mathrm{min}$.

An aixACCT instrument was used to study the electric fielddependent electric polarization. A periodic triangular signal was used for the measurements. A high voltage was obtained using a Trek 609E6 voltage amplifier.

Electron Absorption and Steady-State Photoluminescence. The RT diffuse reflectance spectrum of the powdered sample was measured using a Varian Cary 5E UV-vis-NIR spectrophotometer. Temperature-dependent emission spectra under $266 \mathrm{~nm}$ excitation line from a diode laser were measured with the Hamamatsu photonic multichannel analyzer PMA-12 equipped with a BT-CCD linear image sensor. The temperature of the sample was controlled using a Linkam THMS 600 Heating/Freezing Stage. Time-resolved studies were performed using a femtosecond laser (Coherent Model Libra) as an excitation source.

Single-Crystal X-ray Diffraction. Single-crystal X-ray diffraction was collected at the Xcalibur diffractometer operating with an Atlas $\mathrm{CCD}$ detector and $\mathrm{Mo} \mathrm{K} \alpha$ radiation. Empirical absorption correction using spherical harmonics, implemented in the SCALE3 ABSPACK scaling algorithm, was done using CrysAlis PRO 1.171.38.46 (Rigaku Oxford Diffraction, 2015). $\mathrm{H}$ atom parameters were constrained.

\section{RESULTS AND DISCUSSION}

DSC and TGA. The DSC measurements show the presence of heat anomalies at 342 and $329 \mathrm{~K}$ during heating and cooling, respectively (Figures 1 and S2). This anomaly is symmetric, suggesting a first-order character of the phase transition. The associated changes in enthalpy $\Delta H$ and entropy $\Delta S$ are about $0.97 \mathrm{~kJ} \mathrm{~mol}^{-1}$ and $2.7 \mathrm{~J} \mathrm{~mol}^{-1} \mathrm{~K}^{-1}$, respectively. These values are an order of magnitude smaller than those reported for $\mathrm{MHyPbBr}_{3}\left(9.9 \mathrm{~kJ} \mathrm{~mol}^{-1}\right.$ and $\left.25.3 \mathrm{~J} \mathrm{~mol}^{-1} \mathrm{~K}^{-1}\right){ }^{23}$ For an order-disorder transition, $\Delta S=R \ln (N)$, where $R$ is the gas constant and $N$ is the ratio of the number of configurations in the disordered and ordered phases. Thus, for $N=2$, the phasetransition entropy should be 5.74. The small value of $\Delta S$ suggests, therefore, either a small contribution of an orderdisorder process to the phase transition or a displacive character of this transition. When a sample is heated up to $480 \mathrm{~K}$ and cooled to RT, the anomaly due to the phase transition is still observed on cooling but a new anomaly starts to be visible above $470 \mathrm{~K}$. The third experiment with heating up to $500 \mathrm{~K}$ and cooling down to RT shows a clear peak near $490 \mathrm{~K}$ on heating and a lack of heat anomaly near $330 \mathrm{~K}$ on cooling (Figure S2). These data indicate that $\mathbf{M H y P b C l}_{3}$ decomposes near $\sim 490 \mathrm{~K}$.

The TGA plot indicates that $\mathbf{M H y P b C l}_{3}$ decomposes at $\sim 494$ $\mathrm{K}$ (Figure S3). In this plot, a weight loss of $\sim 22.6 \%$ takes place between 494 and $620 \mathrm{~K}$, corresponding to the release of methylhydrazinium chloride (the calculated value is $22.9 \%$ ). On further heating, $\mathrm{PbCl}_{2}$ starts to sublime at $\sim 770 \mathrm{~K}$, and this process ends above $1000 \mathrm{~K}$.

Single-Crystal X-ray Diffraction. $\mathrm{MHyPbCl}_{3}$ crystallizes in the monoclinic polar space group $P 2_{1}$ (phase II) and at $T_{c}=$ $342 \mathrm{~K}$ transforms to orthorhombic $P b 2_{1} m$ (phase I) (Table S1). The transformation from I $\rightarrow$ II is translationengleiche, groupsubgroup related; $P 2_{1}$ is a maximal subgroup of $P b 2_{1} m$. Phase II is isotypic with its bromine analogue $\mathbf{M H y P b B r}_{3}{ }^{23}$ Because of the presence of two inequivalent positions of lead ions that occupy alternating [001] layers, the 3D structure is highly anisotropic, as displayed in Figure $2 \mathrm{~b}$. It is composed of two types of inorganic layers. One layer is built up of moderately distorted $\mathrm{Pb}(1) \mathrm{Cl}_{6}$ octahedra $(\mathrm{Pb}-\mathrm{Cl}$ bonds of $2.803-2.922 \AA$, Table S2) that exhibit out-of-plane tilting along the $a$-axis and inplane tilting along the $c$-axis. The second layer can be regarded as being composed of massively deformed $\mathrm{Pb}(2) \mathrm{Cl}_{6}$ octahedra with $\mathrm{Pb}-\mathrm{Cl}$ bonds of 2.818-3.170 $\AA$ (Table S2). However, one should take note of the ability of the $\mathrm{NH}_{2}{ }^{+}$terminal groups of $\mathrm{MHy}^{+}$cations to form coordination bonds with $\mathrm{Pb}(2)$ metal centers, as pictured in Figure $2 \mathrm{c}$ and $\mathrm{d}$. In II, the $\mathrm{Pb}(2)-\mathrm{NH}_{2}$ bonds are equal to 2.83 and $2.89 \AA$, which is below the maximum limit for this kind of interaction, as established based on the survey of the CCDC database; see Figures S4 and S5 in the Supporting Information. Thus, in $\mathbf{M H y P b C l} \mathbf{P H}_{3}$ the deformation of every second perovskite layer is forced by oversized $\mathrm{MHy}^{+}$ cations that possess the proper (terminal) placement of amine groups so that they can find their place in the $\mathrm{Pb}(2)$ coordination sphere, and this result is consonant with what was observed for $\mathbf{M H y P b B r}_{3}$ recently. ${ }^{23}$ It must be pointed out, however, that the strength of these interactions is greater in $\mathbf{M H y P b C l}{ }_{3}$ compared to that in the bromine analogue, which can be highlighted as follows.

If we consider the $\mathbf{M H y P b B r}_{3}$ analogue, it is evident that all bonds between $\mathrm{MHy}^{+}$and the organic substructure are broken above $T_{c}$, so that the lattice symmetry increases to the regular $P m \overline{3} m$ centrosymmetric space group, which is characterized by free rotation of $\mathrm{MHy}^{+}$cations and a regular perovskite substructure. As a consequence, the octahedral deformation parameters decrease to 0 . By contrast, in $\mathbf{M H y P b C l}$ one notes 
(a) PHASE I, $360 \mathrm{~K}$
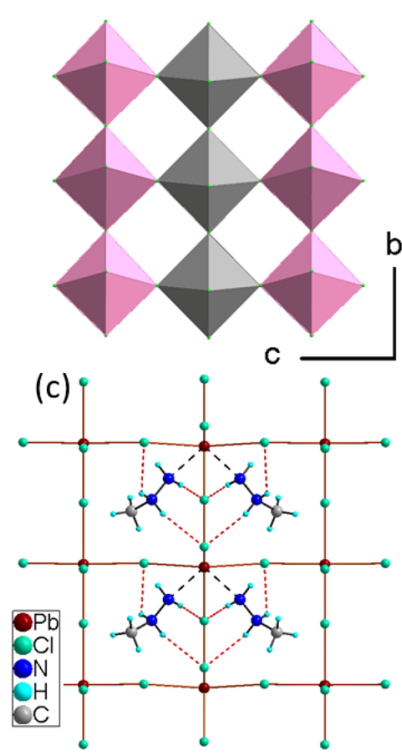

(b) PHASE II, $300 \mathrm{~K}$
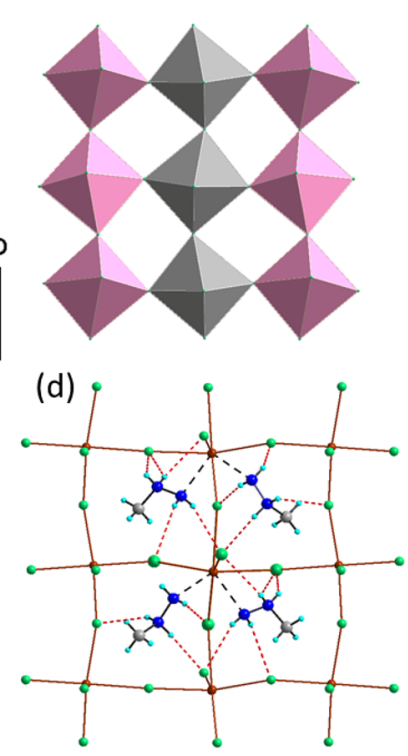

Figure 2. (a, b) Perovskite structures in I and II. The rose color represents $\mathrm{Pb}(1) \mathrm{Cl}_{6}$ octahedra, and gray represents $\mathrm{Pb}(2) \mathrm{Cl}_{6}$. To facilitate comparison with typical 3D lead halide perovskites, the two $\mathrm{N}$ atoms coordinating to $\mathrm{Pb}(2)$ centers are not shown. (c, d) Crystal structures of I and II. Dotted red lines denote hydrogen bonds, and dashed black lines show $\mathrm{Pb}-\mathrm{NH}_{2}$ coordinate interactions.

that the phase transition does not bring such radical changes: neither breaking of $\mathrm{Pb}-\mathrm{NH}_{2}$ bonds occurs nor does the deformation of the lead cations coordination sphere disappear, as was the case of phase I of $\mathbf{M H y P b B r}_{3}$. Indeed, phase I of $\mathbf{M H y P b C l}_{3}$ is still ordered, with clearly discernible perovskite layers that feature deformation parameters comparable to those characterizing phase II. Here, the only exception is the $\Delta d$ parameter that significantly increases for the deformed octahedra (Table 1). Finally, in I, the number of inequivalent lead positions is preserved and the $\mathrm{Pb}(1) \mathrm{Cl}_{6}$ octahedra exhibit in-plane tilt along the $c$-axis. In contrast to phase II, they are located at the mirror planes; thus, the monoclinic distortion vanishes. It is worth adding that stabilities of perovskite phases are often predicted based on the Goldschmidt's tolerance factor (GTF). ${ }^{1}$ To use the concept of GTF for hybrid perovskites, two conditions must be satisfied, i.e., octahedral coordination of metal cations and free rotation of the organic cation. ${ }^{1}$ These conditions are satisfied for the HT cubic phase of $\mathrm{MHyPbBr}_{3}$, for which GTF was $1.03 .{ }^{23}$ However, they are not satisfied for $\mathrm{MHyPbCl}_{3}$, and therefore, the concept of GTF cannot be applied for this compound.

The phase transition arises due to the reorientation of half of the $\mathrm{MHy}^{+}$cations present in the structure combined with a rearrangement of the hydrogen-bond ( $\mathrm{HB})$ network. In both phases, $\mathrm{MHy}^{+}$cations interact with chlorine ligands via $\mathrm{N}-\mathrm{H} \cdots$ $\mathrm{Cl} \mathrm{HBs}$ of medium strength; see the geometrical parameters collected in Table S3. The atom numbering is given in Figure S6. At RT (phase II), there are two inequivalent $\mathrm{MHy}^{+}$cations with all amine hydrogen atoms involved in $\mathrm{N}-\mathrm{H} \cdots \mathrm{Cl}$ bonds. The $\mathrm{N} 1-\mathrm{H} 1 \mathrm{E} \cdots \mathrm{Cl}$ bonding that stands at the origin of the $\mathrm{Pb}(1) \mathrm{Cl}_{6}$ out-of-plane octahedral tilting deserves special attention because it introduces the monoclinic distortion in phase II. In phase I this interaction is not present and the out-ofplane tilting of the $\mathrm{Pb}(1) \mathrm{Cl}_{6}$ octahedra vanishes. Application of AMPLIMODES software ${ }^{25}$ indicates that the order parameter corresponds to lattice instability in the Brillouin zone center of $\Gamma_{2}$ symmetry. Table S4 confirms that octahedral rotation along the $a$-axis contributes significantly to the order parameter. However, shifts of lead atoms also contribute to this displacive mode. On transition to phase I, the number of crystallographically independent $\mathrm{MHy}^{+}$cations is reduced to one, which demands a significant reorientation of $\mathrm{MHy}^{+}$. As a result, new HBs between the middle $\mathrm{NH}_{2}$ groups are formed, and weak bonds between terminal $\mathrm{NH}_{2}{ }^{+}$groups disappear. At the same time, the length of the $\mathrm{Pb}-\mathrm{NH}_{2}$ coordination bond does not change and is equal to $2.88 \AA$.

The earlier-described modifications of the hydrogen-bonding system upon II $\rightarrow$ I phase transition are expected to bring about one important consequence pertaining to the polar properties of these phases. Namely, the rearrangement of the organic substructure causes the dipole moments of $\mathrm{MHy}^{+}$cations to be more closely aligned along the polar $b$-direction (Figure 3 ), which strongly suggests that HT phase I might feature higher spontaneous polarization than RT phase II does. Given that enhancement of polar properties upon structural transition to HT phase is not generally a common phenomenon, in what follows we shall verify this crystallography-based guess using SHG measurements in a wide temperature range.

Temperature-Resolved SHG and Bistability of Nonlinear Response between Two SHG-Active States. Crystallographic studies strongly point to the polar nature of both phases I and II of $\mathbf{M H y P b C l}_{3}$, with the former one presumably featuring more polar character. To test this hypothesis, and to collect broader evidence on the acentricity

Table 1. Comparison of Selected Geometrical Parameters for $\mathrm{MHyPbCl}_{3}$ and Its Analogue, $\mathrm{MHyPbBr}_{3}{ }^{a}$

\begin{tabular}{|c|c|c|c|c|c|c|c|c|c|c|}
\hline name & $\mathrm{Ph}$. & symm. & $T(\mathrm{~K})$ & c.a. & $\Delta d \times 10^{-5}$ & $\sigma^{2}(\operatorname{deg})$ & $\mathrm{X}-\mathrm{Pb}-X_{\text {cis }}(\mathrm{deg})$ & $\mathrm{X}-\mathrm{Pb}-\mathrm{X}_{\mathrm{tr}}(\mathrm{deg})$ & $\mathrm{Pb}-\mathrm{X}-\mathrm{Pb}(\mathrm{deg})$ & $\mathrm{Pb}-\mathrm{NH}_{2}(\AA)$ \\
\hline $\mathrm{MHyPbBr}_{3}$ & I & $P m \overline{3} m$ & 430 & $\mathrm{~Pb}$ & 0 & 0 & 90 & 180 & $\begin{array}{l}180 \\
180^{b}\end{array}$ & \\
\hline & II & $P 2_{1}$ & 293 & $\begin{array}{l}\mathrm{Pb} 1 \\
\mathrm{~Pb} 2\end{array}$ & $\begin{array}{l}5.5 \\
112\end{array}$ & $\begin{array}{l}14 \\
301\end{array}$ & $\begin{array}{l}82-96 \\
71-132\end{array}$ & $\begin{array}{l}172-177 \\
145-157\end{array}$ & $\begin{array}{l}168-172 \\
145-151 \\
157-170^{b}\end{array}$ & $\begin{array}{l}2.915(1) \\
3.044(1)\end{array}$ \\
\hline $\mathrm{MHyPbCl}_{3}$ & I & $P b 2_{1} m$ & 360 & $\begin{array}{l}\mathrm{Pb} 1 \\
\mathrm{~Pb} 2\end{array}$ & $\begin{array}{l}8 \\
146\end{array}$ & $\begin{array}{l}14 \\
302\end{array}$ & $\begin{array}{l}81-96 \\
71-133\end{array}$ & $\begin{array}{l}170-176 \\
143-157\end{array}$ & $\begin{array}{l}170-175 \\
143-154 \\
163\end{array}$ & $2.883(1)$ \\
\hline & II & $P 2_{1}$ & 300 & $\begin{array}{l}\mathrm{Pb} 1 \\
\mathrm{~Pb} 2\end{array}$ & $\begin{array}{l}22 \\
114\end{array}$ & $\begin{array}{l}21 \\
314\end{array}$ & $\begin{array}{l}80-97 \\
81-133\end{array}$ & $\begin{array}{l}170-173 \\
145-156\end{array}$ & $\begin{array}{l}166-170 \\
145-150 \\
155-169^{b}\end{array}$ & $\begin{array}{l}2.830(1) \\
2.893(1)\end{array}$ \\
\hline
\end{tabular}

${ }^{a} \mathrm{X}$ : $\mathrm{Br}, \mathrm{Cl}$. c.a., central atom; $\Delta d$, bond length distortion; $\sigma^{2}$, octahedral angle variance. ${ }^{24} b^{b}$ Angles between layers. 
ENHACEMENT OF SPONTANEOUS POLARIZATION AT HIGH TEMPERATURE!
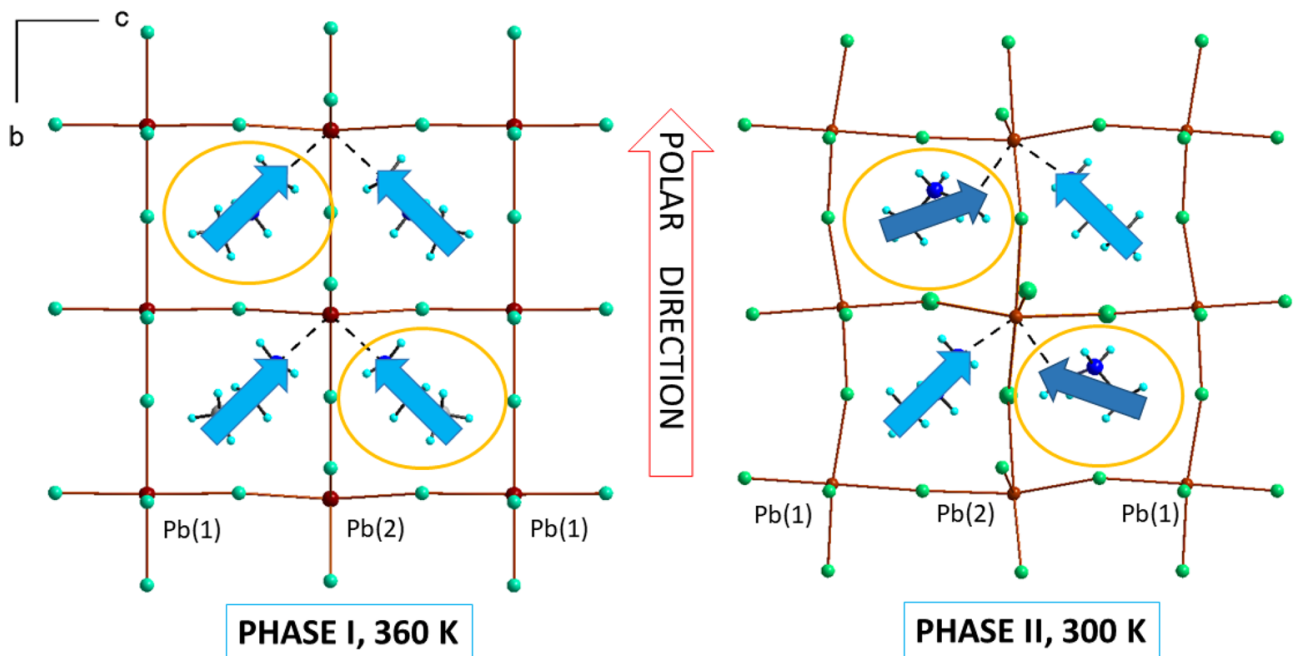

Figure 3. Location of $\mathrm{MHy}^{+}$dipole moments in both phases. Phase transition leads to rotation of every second $\mathrm{MHy}^{+}$and reorganization of the dipoles setting. The polar properties are boosted at high temperature.

of these phases, the temperature-resolved SHG measurements have been performed using $1300 \mathrm{~nm}$ femtosecond laser pulses.

Figure 4a provides a picture of the SHG signal evolution $\left(\lambda_{\operatorname{maxSHG}}=650 \mathrm{~nm}\right)$ for $\mathrm{MHyPbCl}_{3}$ collected for heating and cooling runs (measurement range of $298-373 \mathrm{~K}$ ), whereas in Figure S7 are collected the corresponding experimental spectra. During the heating run, the SHG signal gradually decreases up to $\sim 337 \mathrm{~K}$, at which point it abruptly rises by $\sim 50 \%$ owing to the phase transition to $I$. With a further increase of temperature, the SHG signal decreases at an approximately constant rate up to $373 \mathrm{~K}$. Upon cooling of the sample, one sees that the SHG response remains at the same level down to $332 \mathrm{~K}$, where it drops by ca. $40 \%$. Below $332 \mathrm{~K}$, no substantial changes in SHG intensity are observed. The noticeable downward drift of integral intensities of SHG with temperature increase is due to the superposition of two effects: the increase of thermal motions of structure components and some degree of photochemical bleaching of the sample. Note that upon cooling this drift is much less pronounced since the freezing of thermal motions roughly compensates irradiation-induced deterioration of SHG activity. Taken together, these results provide an ample evidence that both phases feature noncentrosymmetric setting, and more importantly, demonstrate that phase II is more SHG-active than I, confirming what was deduced from X-ray diffraction data.

Next, to quantify the relative strength of the second harmonic of radiation generated by phase II, the Kurtz-Perry powder test $^{26}$ was performed using $1300 \mathrm{~nm}$ pumping at $298 \mathrm{~K}$ on $\mathrm{MHyPbCl}_{3}$ sample sieved into $125-177 \mu \mathrm{m}$ particle-size range. By comparison of the integral intensity of the SHG signal to the one collected for the KDP powder of the same particle size (Figure S8), we found that phase II of $\mathbf{M H y P b C l}{ }_{3}$ offers an SHG response approximately equal to 0.03 that of $\mathrm{KDP}$. The relative efficiency of SHG produced by $\mathbf{M H y P b C l}_{3}$ is 6 times lower when compared to the recently characterized $\mathbf{M H y P b B r}_{3}$ analogue (0.18 that of $\mathrm{KDP}$, also measured at $298 \mathrm{~K}$ using $1300 \mathrm{~nm}$ excitation). ${ }^{23}$

It should be stressed that $\mathbf{M H y P b C l}_{3}$ is the second example of 3D lead halide perovskite comprising corner-sharing $\mathrm{PbX}_{6}$ octahedra that has noncentrosymmetric lattice at RT, a feature that is not present in archetypal $3 \mathrm{D}$ analogues comprising $\mathrm{MA}^{+}$,

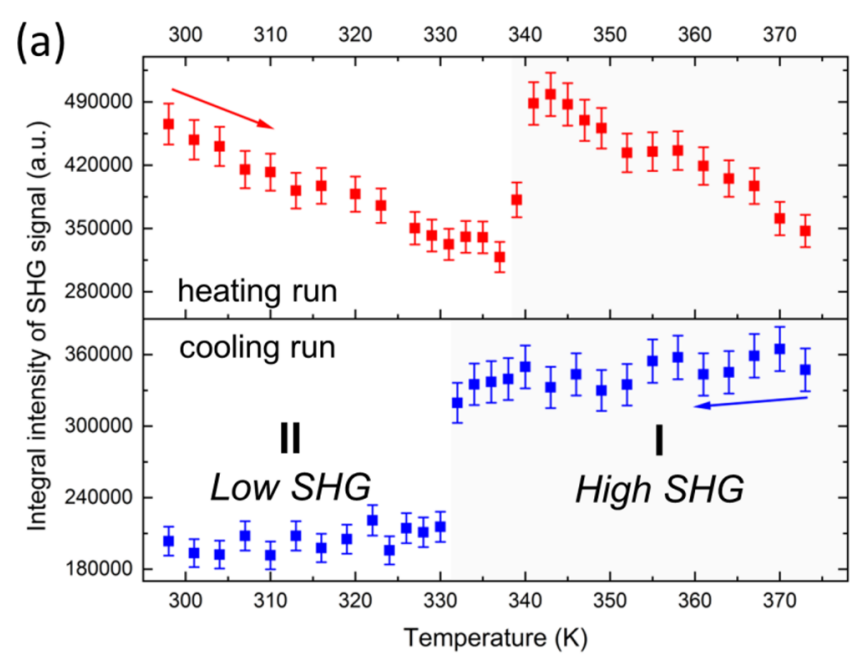

(b)

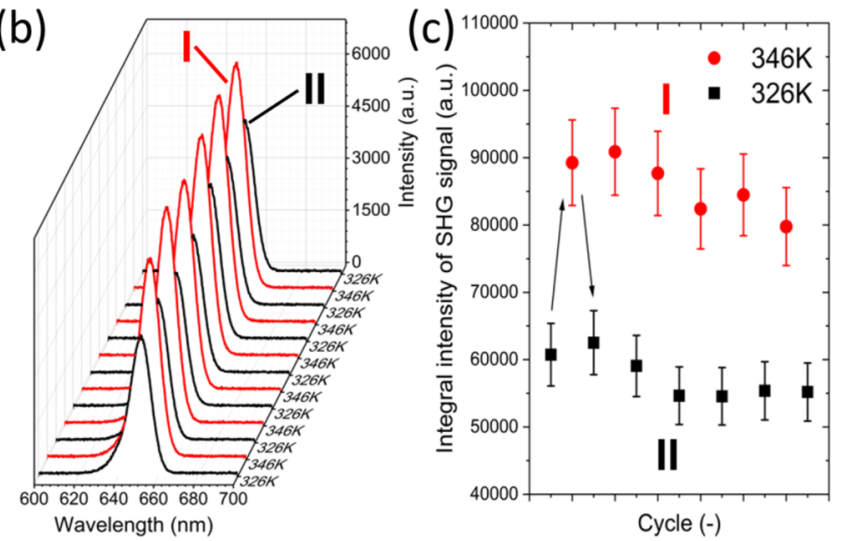

Figure 4. (a) Plots of integral intensities of SHG signal of $\mathbf{M H y P b C l}_{3}$ for heating run (red squares) and cooling run (blue squares). (b) Experimental spectra of SHG signal obtained during switching experiment. Spectra were collected at $326 \mathrm{~K}$ (black traces) and $346 \mathrm{~K}$ (red traces). (c) Plot of integral intensities of SHG collected during switching experiment at $326 \mathrm{~K}$ (black squares) and $346 \mathrm{~K}$ (red circles).

$\mathrm{FA}^{+}$, or $\mathrm{Cs}^{+}$cations. Indeed, until the discovery of $\mathrm{MHyPbBr}_{3}$ perovskite, the only reported noncentrosymmetric lead halide 
perovskites were of layered type (e.g., Ruddelsden-Popper phases $)^{L /, L \gamma}$ and recently found $3 \mathrm{D}$ perovskite containing both corner- and edge-sharing $\mathrm{PbCl}_{6}$ octahedra. ${ }^{15}$ The presence of the methylhydrazinium cation in both compounds therefore raises the question of whether their noncentrosymmetric structure is just an interesting coincidence or there really is some specific structural factor intrinsic to $\mathrm{MHy}^{+}$ions that favors the formation of noncentrosymmetric perovskite phases. Bearing in mind that two examples of noncentrosymmetric 3D perovskites are far too few to make any broad generalizations, we suggest here that the formation of acentric 3D lead halide perovkites may be connected with high framework distortion, induced by the templating effect of an "oversized" cation such as $\mathrm{MHy}^{+}$. This hypothesis will seek experimental verification in the near future.

Recent years have witnessed a dramatic increase of interest in hybrid compounds that exhibit switchable physicochemical properties under the influence of external stimuli such as temperature or pressure. One of the emerging directions in that field is the design of materials that feature bistability of the NLO response (most commonly of SHG output) that could be achieved at around or above RT. Given that the increase of temperature generally favors structural disorder and structural phase transitions increasing crystal symmetry, frequently resulting in transition from noncentrosymmetric to centrosymmetric space groups, the vast majority of available quadratic NLO switches operate between two logic states: SHG-on and SHG-off, corresponding to temperatures below and above $T_{\mathcal{O}}$ respectively. ${ }^{29-46}$ On the other hand, SHG switches that employ two different SHG-active states are a rarity, but still, the hightemperature phases usually feature lower SHG response than low-temperature ones. ${ }^{47}$ Keeping in mind the unusual enhancement of the $\mathbf{M H y P b C l}_{3}$ SHG response that occurs upon II $\rightarrow$ I phase transition, as evidenced by the TR-SHG results (Figure $4 \mathrm{a})$, this $3 \mathrm{D}$ perovskite seems to be the right candidate for demonstration of NLO switching in which SHG is switched between RT low-SHG state and HT high-SHG state.

Figure $4 \mathrm{~b}$ presents a set of experimental spectra of secondharmonic response obtained by irradiation of $\mathbf{M H y P b C l}_{3}$ with $1300 \mathrm{~nm}$ femtosecond laser pulses at two temperatures: 326 and $346 \mathrm{~K}$, at which phases II and I are stable, respectively. By toggling between these temperature points over several cycles, we were able to systematically increase upon heating and decrease upon cooling the SHG response of $\mathbf{M H y P b C l}_{3}$. Integral intensities of obtained SHG signals are displayed in Figure $4 \mathrm{c}$, and they show that the SHG response at $346 \mathrm{~K}$ is $\sim 40-45 \%$ higher than that at the preceding (326 K) temperature point, which is in agreement with what was observed in TR-SHG measurements. Accordingly, $\mathbf{M H y P b C l}_{3}$ may be considered the first example of quadratic NLO switching between two SHG-active states, with high $T_{\mathrm{c}}$ of $337 \mathrm{~K}$, in which the HT phase corresponds to the high-SHG state. Note that the enhancement of the SHG response upon transition to the HT phase was only recently reported in the literature, yet material featuring this property operated well below RT (235 K); also, the switching behavior itself has not been proved directly. ${ }^{48}$

A fair description of $\mathbf{M H y P b C l}_{3}$ quadratic NLO switching properties also requires highlighting the weak points of this material that limit its practical use. Probably the most important drawback is some degree of sensitivity of that material to laser irradiation; this issue finds its reflection in TR-SHG data, visible as the downward drift of SHG intensities, as discussed earlier. Less-pronounced photochemical bleaching of the sample is also apparent in single-temperature measurements; see Figure 4c. From the practical point of view, the contrast of SHG response also is important. Whereas "traditional" SHG switches operating between SHG-on and SHG-off states reach contrast rates as high as hundreds, the NLO switch based on $\mathbf{M H y P b C l}_{3}$ features the contrast (defined as the ratio of integral intensity of the secondharmonic signals of high-SHG to low-SHG crystal phases) of ca. 1.5. Accordingly, although $\mathbf{M H y P b C l}_{3}$ itself reveals an exotic type of SHG bistability, there is still a lot of room for improvements of properties of similar NLO switches employing two SHG-active states.

Electrical Measurements. We performed dielectric and electrical measurements of $\mathbf{M H y P b C l}_{3}$ to further investigate the structural phase transition and properties of phase I. The temperature dependences of the real $\varepsilon^{\prime}$ and imaginary $\varepsilon^{\prime \prime}$ parts of the complex dielectric permittivity $\varepsilon^{*}=\varepsilon^{\prime}-i \varepsilon^{\prime \prime}$ of the $\mathbf{M H y P b C l}_{3}$ pellet sample are presented in parts a and $\mathrm{b}$ of Figure 5 , respectively. A small anomaly of $\varepsilon^{\prime}$ can be observed at $\sim 331 \mathrm{~K}$
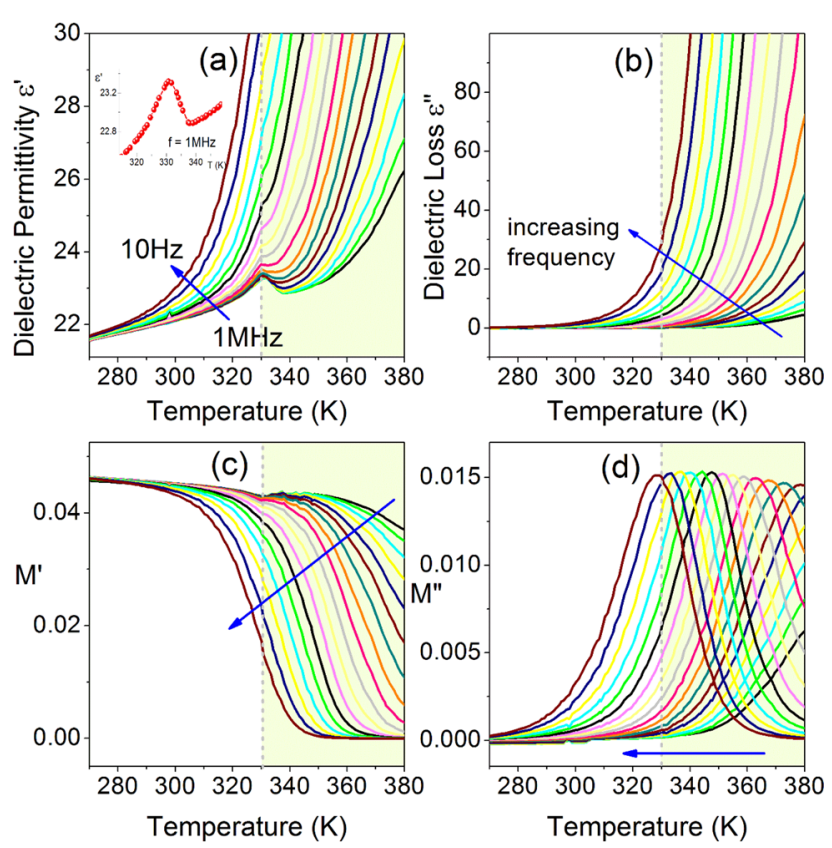

Figure 5. Temperature dependence of the (a) dielectric permittivity, (b) dielectric loss, (c) real $\mathrm{M}^{\prime}$, and (d) imaginary $\mathrm{M}^{\prime \prime}$ components of electric modulus spectra as a function of temperature of the $\mathbf{M H y P b C l}_{3}$ pellet measured on heating. The representative curves are plotted in frequency decades between $10 \mathrm{~Hz}$ and $1 \mathrm{MHz}$. The changes of dielectric permittivity for $1 \mathrm{MHz}$ were enlarged and are presented in the inset in (a). Dashed lines correspond to the structural phase-transition temperature.

on heating, indicating a structural phase transition. In contrast to the temperature-resolved SHG results, in which the phase transition had a clear effect on the intensity of the produced second harmonic of radiation, the observed dielectric anomaly leads to a rather subtle change in the $\varepsilon^{\prime}$ value. For this reason, we have not investigated temperature-induced switching of the dielectric permittivity. The overall increase of $\varepsilon^{*}$ with increasing temperature is due to the electrical conductivity. To reduce the effect of the conductivity characteristic for semiconducting compounds at higher temperatures, the modulus representation $\left(\mathrm{M}^{*}=1 / \varepsilon^{*}\right)$ was used (see Figure $5 \mathrm{c}$ and $\left.\mathrm{d}\right){ }^{49}$ The complex modulus representation of data exhibits strong frequencydependent changes, clearly enhanced at high temperatures, with 
a small anomaly owing to the structural phase transition (Figure $5 \mathrm{c}$ ). The peak maximum of $\mathrm{M}^{\prime \prime}$ shifts toward high temperatures with increasing frequency, which indicates that the observed relaxation becomes thermally activated.

The temperature-dependent current of the $\mathbf{M H y P b C l}_{3}$ pellet after poling the sample with $180 \mathrm{~V} / \mathrm{cm}$ electric field is presented in Figure 6. The current increases rapidly with the increase of

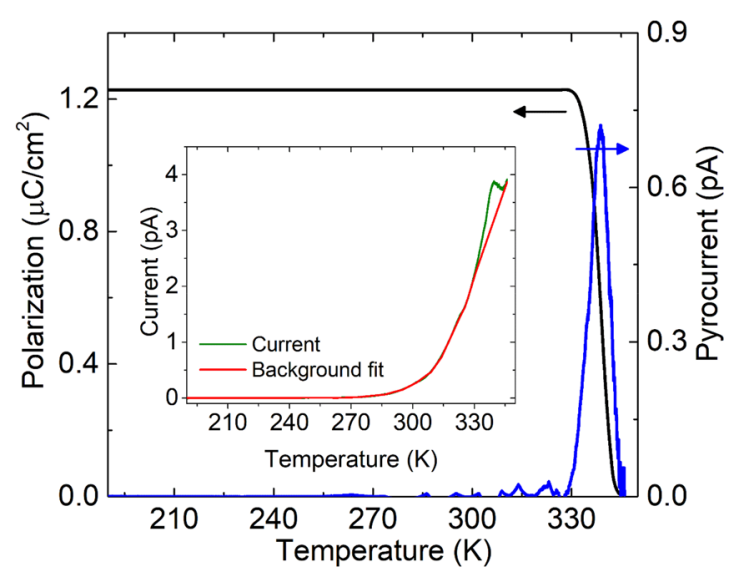

Figure 6. Temperature dependence of the background-corrected pyroelectric current and electric polarization of the $\mathbf{M H y P b C l}_{3}$ pellet measured after poling the sample with $180 \mathrm{~V} / \mathrm{cm}$ electric field. The current prior to the background correction and the exponential background fit are presented in the inset.

temperature, while a phase-transition anomaly is observed at $\sim 339 \mathrm{~K}$ on heating. The pyrocurrent is obtained by subtracting the transition-independent background (inset in Figure 6). The integration of the baseline-corrected pyrocurrent yields the temperature dependence of the electric polarization (Figure 6), which saturates to $1.22 \mu \mathrm{C} / \mathrm{cm}^{2}$. Note that the obtained value of polarization is rather sensitive to the background correction, although it is clearly much higher than in the antipolar $\mathrm{MAPbX}_{3}$ (X $=\mathrm{Cl}, \mathrm{Br}, \mathrm{I})$ family. ${ }^{19}$

The acentricity of both phases and the observed pyroelectric response could indicate a proper ferroelectric behavior of $\mathbf{M H y P b C l}_{3}$. This phenomenon is occasionally encountered in hybrid compounds, ${ }^{50,51}$ although the majority of such compounds are less attractive from the applicational standpoint for improper ferroelectrics. ${ }^{52}$ To check for a proper ferroelectric response, we measured the electric-field dependence of the electric polarization at $323 \mathrm{~K}$ of a single-crystal sample (Figure S9). As can be seen, we did not observe any switching of the electric polarization up to an electric field of $55 \mathrm{kV} / \mathrm{cm}$, demonstrating the absence of proper ferroelectric behavior in $\mathbf{M H y P b C l}_{3}$. We stress that the obtained closed loop originates from a conductivity of the sample, and it is not the ferroelectric hysteresis loop because no switching of the current is observed. ${ }^{51,53}$ The incapability of cation reversible switching by an external electric field in the host-guest system was also observed for metal-organic formate templated by $\mathrm{MHy}^{+}{ }^{54}$ Such results suggest that $\mathrm{MHy}^{+}$hybrid systems may just exhibit improper ferroelectricity. The absence of proper ferroelectric behavior in $\mathbf{M H y P b C l}_{3}$ is consistent with the X-ray diffraction data showing transition from the $P b 2_{1} m$ to the $P 2_{1}$ phase because the $m m 2 F 2$ type transition is not a ferroelectric one. ${ }^{55}$

Optical Properties. The diffuse reflectance spectrum, presented in Figure S10a, shows a band at $373 \mathrm{~nm}$ that can be
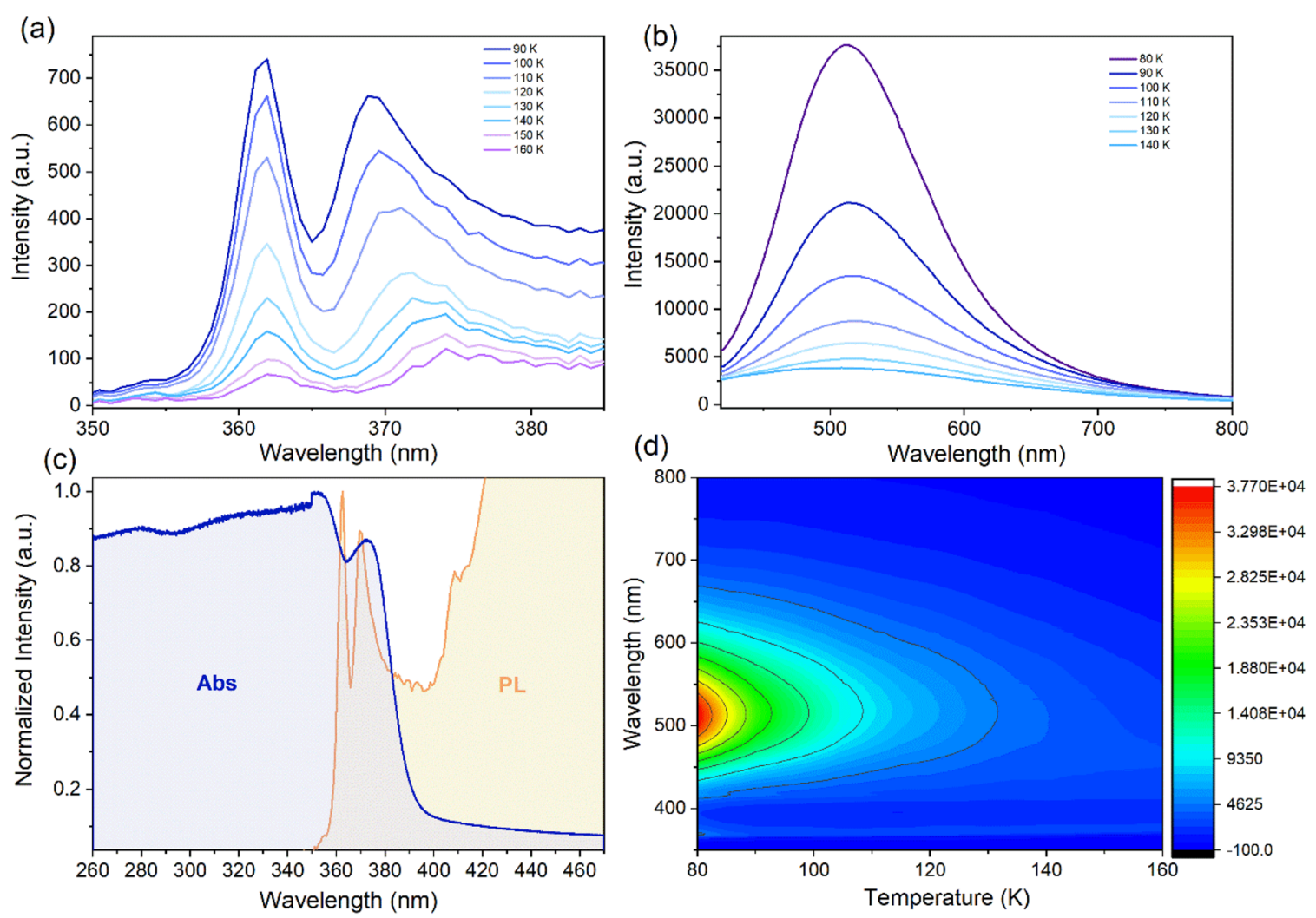

Figure 7. (a, b) Temperature-dependent PL spectra, (c) normalized RT absorption and $90 \mathrm{~K}$ PL spectra, and (d) temperature dependence of bands' intensity (contour map). 
attributed to excitonic absorption. The RT diffuse reflectance spectrum was used to determine the energy bandgap $\left(E_{\mathrm{g}}\right)$ of $\mathrm{MHyPbCl}_{3}$ with the Kubelka-Munk relation, ${ }^{56}$

$$
F(R)=\frac{(1-R)^{2}}{2 R}
$$

where $R$ denotes reflectance. The inset to Figure $S 10 \mathrm{~b}$ shows that the value of $E_{\mathrm{g}}$ in $\mathbf{M H y P b C l}_{3}$ is $\sim 365 \mathrm{~nm}(3.4 \mathrm{eV})$. This value is larger than the $E_{\mathrm{g}}$ values of $\mathrm{MAPbCl}_{3}$ (experimental $2.88-2.94 \mathrm{eV}$, calculated $3.04 \mathrm{eV})^{10,57,58}$ and $\mathrm{FAPbCl}_{3}$ (calculated $3.02 \mathrm{eV}$, experimental $3.00 \mathrm{eV}$ ). ${ }^{58,59}$ Similar behavior was observed also for $\mathrm{MHyPbBr}_{3}$, and it can be attributed to much larger distortion of the $\mathrm{PbCl}_{3}{ }^{-}$framework in $\mathbf{M H y P b C l}_{3}$ than in $\mathrm{MAPbCl}_{3}$ or $\mathrm{FAPbCl}_{3}$.

The low-temperature PL spectrum of $\mathbf{M H y P b C l}_{3}$ shows two weak bands close to the excitonic absorption band: a very narrow P1 band at $362 \mathrm{~nm}($ fwhm $=3.5 \mathrm{~nm})$ and a broader P2 band at $369 \mathrm{~nm}(5.7 \mathrm{~nm})$ (Figure 7). Although Figure 7 suggests that the $362 \mathrm{~nm}$ band corresponds to the $E_{\mathrm{g}}$ and that at $369 \mathrm{~nm}$ corresponds to the excitonic absorption, allowing for assigning them to the interband and exciton recombination, respectively, it should be noticed that the PL band corresponds to $90 \mathrm{~K}$ and the excitonic absorption corresponds to RT. Former studies of isostructural $\mathrm{MHyPbBr}_{3}$ also showed that the narrow PL band observed at $80 \mathrm{~K}$ is blue-shifted by $\sim 11 \mathrm{~nm}$ compared to the RT excitonic absorption, but the RT PL band nearly overlaps with the RT excitonic absorption and can be attributed to a free exciton (FE). ${ }^{23}$ We suppose, therefore, that the P1 band of $\mathrm{MHyPbCl}_{3}$ can also be attributed to $\mathrm{FE}$ recombination, observed for isostructural $\mathrm{MHyPbBr}_{3}$ at $457.9 \mathrm{~nm}$ (fwhm = $3.9 \mathrm{~nm}),{ }^{23}$ for $\mathrm{MAPbCl}_{3}$ at $404 \mathrm{~nm}(3.07 \mathrm{eV}$, fwhm $=11 \mathrm{~nm}),{ }^{51}$ and for $\mathrm{CsPbCl}_{3}$ single crystal near $416 \mathrm{~nm}(2.98 \mathrm{eV}) .{ }^{60}$ The second narrow band was not observed for $\mathrm{MHyPbBr}_{3}$, but it was observed for $\mathrm{MAPbCl}_{3}$ as a weak band at $415 \mathrm{~nm}(2.98 \mathrm{eV}$, fwhm $=24 \mathrm{~nm}) .{ }^{57}$ This band originates most likely from the recombination of photoexcited carriers in surface defects. ${ }^{57}$

In addition to the UV PL, $\mathbf{M H y P b C l}{ }_{3}$ shows the presence of a strong broadband emission at $512 \mathrm{~nm}$ (band P3, fwhm $=125$ $\mathrm{nm}$ ) (Figure 7). Time-resolved measurements at $80 \mathrm{~K}$ under 266 $\mathrm{nm}$ excitation line show that this PL can be best fitted by biexponential decay function with $\tau_{1}=0.065 \mathrm{~ns}$ and $\tau_{2}=0.306 \mathrm{~ns}$ (Figure S11). The lifetimes decrease with increasing temperature (Figure S11). Our recent studies of isostructural $\mathrm{MHyPbBr}_{3}$ also revealed the presence of red-shifted PL that was attributed to bound exciton (BE) recombination. ${ }^{23}$ However, the Stokes shifts of the two broad PL bands were much smaller (27.8 and $47.8 \mathrm{~nm}$ compared to $150 \mathrm{~nm}(\sim 1 \mathrm{eV})$ in $\left.\mathrm{MHyPbCl}_{3}\right)$, and the bands were narrower $(\mathrm{fwhm}=17.3$ and $19.5 \mathrm{~nm}) .{ }^{23}$ Thus, the $\mathrm{P} 3$ band of $\mathrm{MHyPbCl}_{3}$ cannot be attributed to $\mathrm{BE}$ states, but the very large width and Stokes shift suggest that it originates from many energy levels within the bandgap. Because the PL measurements were performed on millimeter-sized single crystals, it is very likely that the broadband emission does not originate from radiative surface states but is an intrinsic property of $\mathbf{M H y P b C l}_{3}$. Therefore, we tentatively assign this broadband emission to self-trapped excition (STEx) states. Weak emission attributed to STEx was observed for $\mathrm{CsPbCl}_{3}$ below $150 \mathrm{~K}$ at $1.9 \mathrm{eV}(653 \mathrm{~nm}),{ }^{60}$ and strong STE-related emission was observed near 500-600 $\mathrm{nm}$ for many two-dimensional lead chlorides with a strongly distorted lead chlorine framework. ${ }^{61,62}$ Interestingly, very similar broadband emission was also reported for three-layered perovskite $\left(\mathrm{CH}_{3} \mathrm{CH}_{2} \mathrm{NH}_{2}\right)_{4} \mathrm{~Pb}_{3} \mathrm{Cl}_{10}$, which contains inorganic layers composed of two types of $\mathrm{PbCl}_{6}$ octahedra, one strongly distorted (the distortion parameter $\Delta d=45.09 \times 10^{-4}$ ) and one less distorted $\left(\Delta d=13.15 \times 10^{-4}\right) .{ }^{63}$ Although $\mathbf{M H y P b C l}_{3}$ does not possess organic layers separating inorganic slabs, like $\left(\mathrm{CH}_{3} \mathrm{CH}_{2} \mathrm{NH}_{2}\right)_{4} \mathrm{~Pb}_{3} \mathrm{Cl}_{10}$, which contains layers of ethylammonium cations, it resembles this perovskite in a sense that its structure also consists of two inorganic layers, one weakly distorted $\left(\Delta d=2.2 \times 10^{-4}\right)$ and the other strongly deformed $\left(\Delta d=11.4 \times 10^{-4}\right)$. Thus, the presence of the broadband whitelight emission in $\mathbf{M H y P b C l}_{3}$ can most likely be attributed to the very large structural distortion and polar nature of this compound. The presence of less-broad and smaller Stokesshifted bands for $\mathrm{MHyPbBr}_{3}{ }^{23}$ is consistent with smaller distortion of its inorganic layers (Table 1).

$\mathrm{PL}$ intensity decreases quickly on heating, and PL is hardly visible already at $180 \mathrm{~K}$ (Figures 7 and S11). On the basis of the collected spectra, CIE (Commission Internationale de l'Eclairage) chromaticity coordinates have been calculated, which allowed for the depiction in Figure 8 of temperature-dependent

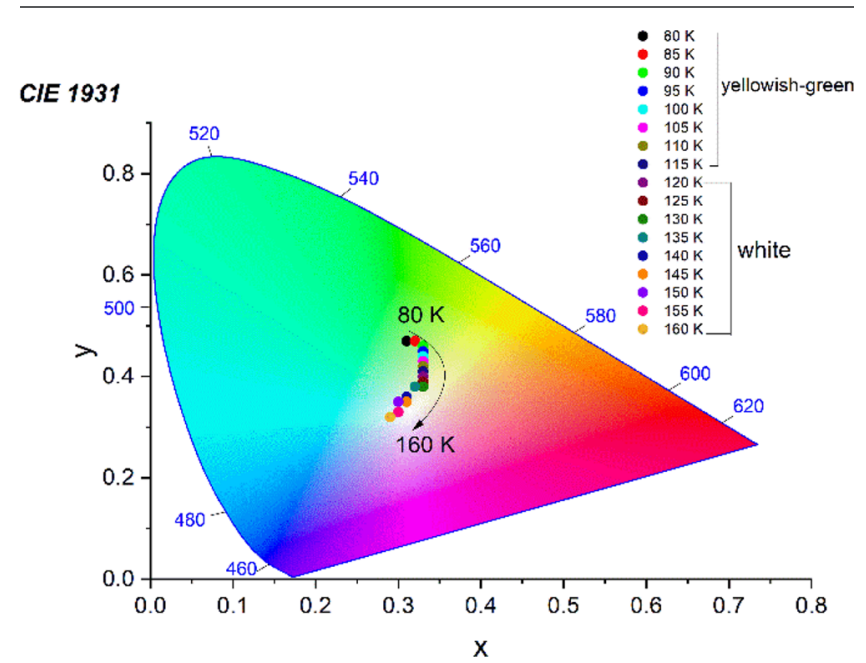

Figure 8. CIE coordinates of $\mathrm{MHyPbCl}_{3}$ at different temperatures.

emission color changes. The color changes from yellowish-green at $80 \mathrm{~K}$ to white above $115 \mathrm{~K}$. For instance, the chromaticity coordinates $(x, y)$ of the white emission change from warmer $(0.33,0.40)$ at $120 \mathrm{~K}$ to colder $(0.30,0.33)$ at $150 \mathrm{~K}$.

\section{CONCLUSIONS}

We report the synthesis of a new all-noncentrosymmetric 3D perovskite, methylhydrazinium lead chloride. The energy bandgap of $\mathbf{M H y P b C l}_{3}(365 \mathrm{~nm}, 3.4 \mathrm{eV})$ is larger than the bandgaps of other known 3D lead chloride perovskites comprising methylammonium $(\sim 2.88-2.94 \mathrm{eV})$ or formamidinium $(\sim 3.0 \mathrm{eV})$. Furthermore, contrary to $\mathrm{FAPbCl}_{3}, \mathrm{MAPbCl}_{3}$, and $\mathrm{CsPbCl}_{3}$, which exhibit narrow excitonic blue $\mathrm{PL}$, $\mathrm{MHyPbCl}_{3}$ shows weak UV and strong broadband white emission. It crystallizes in a polar $P 2_{1}$ structure, while the earlier mentioned 3D lead chlorides all possess centrosymmetric structures. TR-SHG measurements also revealed that the second-harmonic response is enhanced for the high-temperature $P b 2_{1} m$ phase compared to the RT $P 2_{1}$ phase. This intriguing property of $\mathrm{MHyPbCl}_{3}$ has been employed to demonstrate an unprecedented kind of quadratic nonlinear optical switching in which the second-harmonic response is switched between the RT low-SHG state and the HT high-SHG state. 
Different optical and electrical characteristics of $\mathbf{M H y P b C l}$, compared to the known $3 \mathrm{D}$ analogues, can be attributed to the presence of two perovskite layers, one weakly distorted and the other strongly deformed. Our studies prove that exceptionally large distortion of the $\mathrm{Pb}(2) \mathrm{Cl}_{6}$ octahedral layer is forced by the large size of the methylhydrazinium cations and the ability of $\mathrm{NH}_{2}^{+}$terminal groups of methylhydrazinium cations to form coordinate covalent bonds with $\mathrm{Pb}^{2+}$ metal centers.

We also show that $\mathbf{M H y P b C l}_{3}$ undergoes a structural phase transition at $342 \mathrm{~K}$ into another polar $\mathrm{Pb2}_{1} \mathrm{~m}$ phase, which possesses even more distorted $\mathrm{Pb}(2) \mathrm{Cl}_{6}$ octahedra but still ordered $\mathrm{MHy}^{+}$cations. The increase of SHG activity in the $P b 2_{1} m$ phase was attributed to the specific reorientation of $\mathrm{MHy}^{+}$, resulting in more parallel setting of the dipoles in respect to the polar $b$-direction. Electrical studies revealed a clear pyroelectric anomaly. However, the switching of the electric polarization could not be observed up to an electric field of 55 $\mathrm{kV} / \mathrm{cm}$, indicating the absence of proper ferroelectric behavior in $\mathrm{MHyPbCl}_{3}$.

\section{ASSOCIATED CONTENT}

\section{SI Supporting Information}

The Supporting Information is available free of charge at https://pubs.acs.org/doi/10.1021/acs.chemmater.0c00973.

Crystal data, selected geometrical and $\mathrm{HB}$ parameters for the studied compound at 300 and $360 \mathrm{~K}$, atomic displacements after the symmetry change from the $P b 2_{1} m$ to the $P 2_{1}$ phase, powder XRD pattern, DSC traces, TGA plot, histogram representing length of $\mathrm{Pb}-$ $\mathrm{NH}_{2}$ coordinate bonds, crystal structures and $\mathrm{HB}$ interactions, SHG traces, electric-field dependence of the electric polarization and current, diffuse reflectance spectrum, and temperature-dependent decay times (PDF)

Structure at $300 \mathrm{~K}(\mathrm{CIF})$

Structure at $360 \mathrm{~K}$ (CIF)

\section{AUTHOR INFORMATION}

\section{Corresponding Author}

Mirosław Mączka - Institute of Low Temperature and Structure Research, Polish Academy of Sciences, 50-950 Wroctaw 2, Poland; (1) orcid.org/0000-0003-2978-1093; Phone: +48713954161; Email: m.maczka@int.pan.wroc.pl; Fax: +48713441029

\section{Authors}

Anna Gagor - Institute of Low Temperature and Structure Research, Polish Academy of Sciences, 50-950 Wroctaw 2, Poland

Jan K. Zarȩba - Advanced Materials Engineering and Modeling Group, Faculty of Chemistry, Wroclaw University of Science and Technology, 50-370 Wroctaw, Poland; () orcid.org/00000001-6117-6876

Dagmara Stefanska - Institute of Low Temperature and Structure Research, Polish Academy of Sciences, 50-950 Wroctaw 2, Poland

Marek Drozd - Institute of Low Temperature and Structure Research, Polish Academy of Sciences, 50-950 Wroctaw 2, Poland

Sergejus Balciunas - Faculty of Physics, Vilnius University, LT10222 Vilnius, Lithuania

Mantas Simenas - Faculty of Physics, Vilnius University, LT10222 Vilnius, Lithuania; $\odot$ orcid.org/0000-0002-2733-2270
Juras Banys - Faculty of Physics, Vilnius University, LT-10222 Vilnius, Lithuania

Adam Sieradzki - Department of Experimental Physics, Wroctaw University of Science and Technology, 50-370 Wrocław, Poland; (1) orcid.org/0000-0003-4136-5754

Complete contact information is available at:

https://pubs.acs.org/10.1021/acs.chemmater.0c00973

\section{Notes}

The authors declare no competing financial interest.

\section{ACKNOWLEDGMENTS}

This work has received funding from the Research Council of Lithuania (LMTLT), Agreement no. S-MIP-19-4. J.K.Z. acknowledges financial support from the Faculty of Chemistry, Wrocław University of Science and Technology. J.K.Z. is supported by the Foundation for Polish Science (FNP).

\section{REFERENCES}

(1) Saparov, B.; Mitzi, D. B. Organic-Inorganic Perovskites: Structural Versatility for Functional Materials Design. Chem. Rev. 2016, 116, 4558-4596.

(2) Li, W.; Wang, Z.; Deschler, F.; Gao, S.; Friend, R. H.; Cheetham, A. K. Chemically Diverse and Multifunctional Hybrid OrganicInorganic Perovskites. Nature Rev. Mater. 2017, 2, 16099.

(3) Zhao, X.; Ng, J. D. A.; Friend, R. H.; Tan, Z.-K. Opportunities and Challenges in Perovskite Light-Emitting Devices. ACS Photonics 2018, 5, 3866-3875.

(4) Hoefler, S. F.; Trimmel, G.; Rath, T. Progress on Lead-Free Metal Halide Perovskites for Photovoltaic Applications: a Review. Monatsh. Chem. 2017, 148, 795-826.

(5) Quan, L. N.; Rand, B. P.; Friend, R. H.; Mhaisalkar, S. G.; Lee, T.W.; Sargent, E. H. Perovskites for Next-Generation Optical Sources. Chem. Rev. 2019, 119, 7444-7477.

(6) Wang, F.; Cao, Y.; Chen, C.; Chen, Q.; Wu, X.; Li, X.; Qin, T.; Huang, W. Materials Toward the Upscaling of Perovskite Solar Cells: Progress, Challenges, and Strategies. Adv. Funct. Mater. 2018, 28, 1803753.

(7) Huang, H.; Polavarapu, L.; Sichert, J. A.; Susha, A. S.; Urban, A. S.; Rogach, A. I. Coloidal Lead Halide Perovskite Nanocrystals: Synthesis, Optical Properties and Applications. NPG Asia Mater. 2016, 8, No. e328.

(8) Mykhaylyk, V. B.; Kraus, H.; Saliba, M. Bright and Fast Scintillation of Organolead Perovskite $\mathrm{MAPbBr}_{3}$ at Low Temperatures. Mater. Horiz. 2019, 6, 1740-1747.

(9) Adinolfi, V.; Ouellette, O.; Saidaminov, M. I.; Walters, G.; Abdelhady, A. L.; Bakr, O. M.; Sargent, E. H. Fast and Sensitive Solution-Processed Visible-Blind Perovskite UV Photodetectors. Adv. Mater. 2016, 28, 7264-7268.

(10) Maculan, G.; Sheikh, A. D.; Abdelhady, A. L.; Saidaminov, M. I.; Haque, M. A.; Murali, B.; Alarousu, E.; Mohammed, O. F.; Wu, T.; Bakr, O. M. $\mathrm{CH}_{3} \mathrm{NH}_{3} \mathrm{PbCl}_{3}$ Single Crystals: Inverse Temperature Crystallization and Visible-Blind UV Photodetector. J. Phys. Chem. Lett. 2015, 6, 3781-3786.

(11) D’Annibale, A.; Panetta, R.; Tarquini, O.; Colapietro, M.; Quaranta, S.; Cassetta, A.; Barba, L.; Chita, G.; Latini, A. Synthesis, Physico-Chemical Characterization and Structure of the Elusive Hydroxylammonium Lead IodidePerovskite $\mathrm{NH}_{3} \mathrm{OHPbI}_{3}$. Dalton Trans. 2019, 48, 5397-5407.

(12) Campbell, E. V.; Dick, B.; Rheingold, A. L.; Zhang, C.; Liu, X.; Vardeny, Z. V.; Miller, J. S. Structures of a Complex Hydrazinium Lead Iodide, $\left(\mathrm{N}_{2} \mathrm{H}_{5}\right)_{15} \mathrm{~Pb}_{3} \mathrm{I}_{21}$, Possessing $\left[\mathrm{Pb}_{2} \mathrm{I}_{9}\right]_{5}^{-},\left[\mathrm{PbI}_{6}\right]_{4}{ }^{-}$, and $\mathrm{I}^{-}$Ions and $\alpha$ - and $\beta-\left(\mathrm{N}_{2} \mathrm{H}_{5}\right) \mathrm{PbI}_{3}$. Chem. - Eur. J. 2018, 24, 222-229.

(13) Tian, J.; Cordes, D. B.; Quarti, C.; Beljonne, D.; Slawin, A. M. Z.; Zysman-Colman, E.; Morrison, F. D. Stable 6H Organic-Inorganic Hybrid Lead Perovskite and Competitive Formation of $6 \mathrm{H}$ and $3 \mathrm{C}$ 
Perovskite Structure with Mixed A Cations. ACS Appl. Energy Mater. 2019, 2, 5427-5437.

(14) Seth, C.; Khushalani, D. Non-Perovskite Hybrid Material, Imidazolium Lead Iodide, with Enhanced Stability. ChemNanoMat 2019, 5, 85-91.

(15) Zhang, H.-Y.; Song, X. J.; Cheng, H.; Zeng, Y.-L.; Zhang, Y.; Li, P.-F.; Liao, W.-Q.; Xiong, R.-G. A Three-Dimensional Lead Halide Perovskite-Related Ferroelectric. J. Am. Chem. Soc. 2020, 142, 46044608.

(16) Govinda, S.; Kore, B. P.; Swain, D.; Hossain, A.; De, C.; Guru Row, T. N.; Sarma, D. D. Critical Comparison of FAPbX ${ }_{3}$ and $\mathrm{MAPbX}_{3}$ $(\mathrm{X}=\mathrm{Br}$ and $\mathrm{Cl})$ : How Do They Differ. J. Phys. Chem. C 2018, 122, 13758-13766.

(17) Fabini, D. H.; Stoumpos, C. C.; Laurita, G.; Kaltzoglou, A.; Kontos, A. G.; Falaras, P.; Kanatzidis, M. G.; Seshadri, R. Reentrant Structural and Optical Properties and Large Positive Thermal Expansion in Perovskite Formamidinium Lead Iodide. Angew. Chem., Int. Ed. 2016, 55, 15392-15396.

(18) Yang, B.; Ming, W.; Du, M.-H.; Keum, J. K.; Puretzky, A. A.; Rouleau, C. M.; Huang, J.; Geohegan, D. B.; Wang, X.; Xiao, K. RealTime Observation of Order-Disorder Transformation of Organic Cations Induced Phase Transition and Anomalous Photoluminescence in Hybrid Perovskites. Adv. Mater. 2018, 30, 1705801.

(19) Simenas, M.; Balciunas, S.; Maczka, M.; Banys, J.; Tornau, E. E. Exploring the Antipolar Nature of Methylammonium Lead Halides: A Monte Carlo and Pyrocurrent Study. J. Phys. Chem. Lett. 2017, 8, 49064911.

(20) Trots, D.; Myagkota, S. High-temperature Structural Evolution of Caesium and Rubidium Triiodoplumbates. J. Phys. Chem. Solids 2008, 69, 2520-2526.

(21) Mączka, M.; Gạgor, A.; Ptak, M.; Paraguassu, W.; Da Silva, T. A.; Sieradzki, A.; Pikul, A. Phase Transitions and Coexistence of Magnetic and Electric Orders in the Methylhydrazinium Metal Formates. Chem. Mater. 2017, 29, 2264-2275.

(22) Mączka, M.; Ptak, M.; Ga̧gor, A.; Stefańska, D.; Sieradzki, A. Layered Lead Iodide of [Methylhydrazinium $]_{2} \mathrm{PbI}_{4}$ with a Reduced Band Gap: Thermochromic Luminescence and Switchable Dielectric Properties Triggered by Structural Phase Transitions. Chem. Mater. 2019, 31, 8563-8575.

(23) Mączka, M.; Ptak, M.; Gągor, A.; Stefańska, D.; Zaręba, J. K.; Sieradzki, A. Methylhydrazinium Lead Bromide: Noncentrosymmetric Three-Dimensional Perovskite with Exceptionally Large Framework Distortion and Green Photoluminescence. Chem. Mater. 2020, 32, 1667-1673.

(24) Fleet, M. E. Distortion Parameters for Coordination Polyhedra. Mineral. Mag. 1976, 40, 531-533.

(25) Perez-Mato, J. M.; Orobengoa, D.; Aroyo, M. I. Mode Crystallography of Distorted Structures. Acta Crystallogr., Sect. A: Found. Crystallogr. 2010, 66, 558-590.

(26) Kurtz, S. K.; Perry, T. T. A Powder Technique for the Evaluation of Nonlinear Optical Materials. J. Appl. Phys. 1968, 39, 3798-3813.

(27) Stoumpos, C. C.; Cao, D. H.; Clark, D. J.; Young, J.; Rondinelli, J. M.; Jang, J. I.; Hupp, J. T.; Kanatzidis, M. G. Ruddlesden-Popper Hybrid Lead Iodide Perovskite 2D Homologous Semiconductors. Chem. Mater. 2016, 28 (8), 2852-2867.

(28) Zhang, Q.; Solanki, A.; Parida, K.; Giovanni, D.; Li, M.; Jansen, T. L. C.; Pshenichnikov, M. S.; Sum, T. C. Tunable Ferroelectricity in Ruddlesden-Popper Halide Perovskites. ACS Appl. Mater. Interfaces 2019, 11, 13523-13532.

(29) Tao, K.; Wu, Z.; Han, S.; Zhang, J.; Ji, C.; Wang, Y.; Zhang, W.; Luo, J.; Sun, Z. Switchable Behaviors of Quadratic Nonlinear Optical Properties Originating from Bi-Step Phase Transitions in a MoleculeBased Crystal. J. Mater. Chem. C 2018, 6, 4150-4155.

(30) Chen, X.-G.; Zhang, Y.-Z.; Sun, D.-S.; Gao, J.-X.; Hua, X.-N.; Liao, W.-Q. Above Room-Temperature Dielectric and Nonlinear Optical Switching Materials Based on $\left[\left(\mathrm{CH}_{3}\right)_{3} \mathrm{~S}\right]_{2}\left[\mathrm{MBr}_{4}\right](\mathrm{M}=\mathrm{Cd}, \mathrm{Mn}$ and Zn). Dalton Trans. 2019, 48, 11292-11297.
(31) Zhang, H.-Y.; Lu, S.-Q.; Chen, X.; Xiong, R.-G.; Tang, Y.-Y. The First High-Temperature Multiaxial Ferroelectric Host-Guest Inclusion Compound. Chem. Commun. 2019, 55, 11571-11574.

(32) Gao, J.-X.; Hua, X.-N.; Chen, X.-G.; Liao, W.-Q. A High Temperature Optic-Electric Duple Switching Organic Ionic Compound: 1,4,7-Triazoniacyclononane Tetrafluoroborate Dichloride. J. Mater. Chem. C 2019, 7, 5348-5352.

(33) Liao, W.-Q.; Gao, J.-X.; Hua, X.-N.; Chen, X.-G.; Lu, Y. Unusual Two-Step Sequential Reversible Phase Transitions with Coexisting Switchable Nonlinear Optical and Dielectric Behaviors in $\left[\left(\mathrm{CH}_{3}\right)_{3} \mathrm{NCH}_{2} \mathrm{Cl}\right]_{2}\left[\mathrm{ZnCl}_{4}\right]$. J. Mater. Chem. C 2017, 5, 11873-11878.

(34) Xu, L.; Gao, J.-X.; Chen, X.-G.; Hua, X.-N.; Liao, W.-Q. A Temperature-Triggered Triplex Bistable Switch in a Hybrid Multifunctional Material: $\left[\left(\mathrm{CH}_{2}\right)_{4} \mathrm{~N}\left(\mathrm{CH}_{2}\right)_{4}\right]_{2}\left[\mathrm{MnBr}_{4}\right]$. Dalton Trans. 2018, 47, 16995-17003.

(35) Zheng, X.; Shi, P.-P.; Lu, Y.; Zhou, L.; Gao, J.-X.; Geng, F.-J.; Wu, D.-H.; Fu, D.-W.; Ye, Q. Dielectric and Nonlinear Optical Dual Switching in an Organic-Inorganic Hybrid Relaxor $\left[\left(\mathrm{CH}_{3}\right)_{3} \mathrm{PCH}_{2} \mathrm{OH}\right]-$ $\left[\mathrm{Cd}(\mathrm{SCN})_{3}\right]$. Inorg. Chem. Front. 2017, 4, 1445-1450.

(36) Wu, Z.; Liu, X.; Ji, C.; Li, L.; Wang, S.; Sun, Z.; Zhang, W.; Peng, Y.; Luo, J. Above-Room-Temperature Switching of Quadratic Nonlinear Optical Properties in a Bi-halide Organic-Inorganic Hybrid. J. Mater. Chem. C 2018, 6, 9532-9536.

(37) Zhang, J.; Han, S.; Liu, X.; Wu, Z.; Ji, C.; Sun, Z.; Luo, J. A Leadfree Perovskite-like Hybrid with Above-Room-Temperature Switching of Quadratic Nonlinear Optical Properties. Chem. Commun. 2018, 54, 5614-5617.

(38) Liu, X.; Ji, C.; Wu, Z.; Li, L.; Han, S.; Wang, Y.; Sun, Z.; Luo, J. $\left[\mathrm{C}_{5} \mathrm{H}_{12} \mathrm{~N}\right] \mathrm{SnCl}_{3}$ : A Tin Halide Organic-Inorganic Hybrid as an AboveRoom-Temperature Solid-State Nonlinear Optical Switch. Chem. - Eur. J. 2019, 25, 2610-2615.

(39) Sun, Z.; Chen, T.; Liu, X.; Hong, M.; Luo, J. Plastic Transition to Switch Nonlinear Optical Properties Showing the Record High Contrast in a Single-Component Molecular Crystal. J. Am. Chem. Soc. 2015, 137, 15660-15663.

(40) Mei, G.-Q.; Zhang, H.-Y.; Liao, W.-Q. A symmetry Breaking Phase Transition-Triggered High-Temperature Solid-State Quadratic Nonlinear Optical Switch Coupled With a Switchable Dielectric Constant in an Organic-Inorganic Hybrid Compound. Chem. Commun. 2016, 52, 11135-11138.

(41) Ji, C.; Sun, Z.; Zhang, S.; Zhao, S.; Chen, T.; Tang, Y.; Luo, J. A Host-Guest Inclusion Compound for Reversible Switching of Quadratic Nonlinear Optical Properties. Chem. Commun. 2015, 51, 2298-2300.

(42) Sun, Z.; Luo, J.; Zhang, S.; Ji, C.; Zhou, L.; Li, S.; Deng, F.; Hong, M. Solid-State Reversible Quadratic Nonlinear Optical Molecular Switch with an Exceptionally Large Contrast. Adv. Mater. 2013, 25, $4159-4163$.

(43) Zhang, Y.; Ye, H.-Y.; Cai, H.-L.; Fu, D.-W.; Ye, Q.; Zhang, W.; Zhou, Q.; Wang, J.; Yuan, G.-L.; Xiong, R.-G. Switchable Dielectric, Piezoelectric, and Second-Harmonic Generation Bistability in a New Improper Ferroelectric above Room Temperature. Adv. Mater. 2014, 26, 4515-4520.

(44) Zhang, W.-Y.; Tang, Y.-Y.; Li, P.-F.; Shi, P.-P.; Liao, W.-Q.; Fu, D.-W.; Ye, H.-Y.; Zhang, Y.; Xiong, R. G. Precise Molecular Design of High- $\mathrm{T}_{\mathrm{c}} 3 \mathrm{D}$ Organic-Inorganic Perovskite Ferroelectric: [MeHdabco]$\mathrm{RbI}_{3}$ (MeHdabco= N-Methyl-1,4-diazoniabicyclo[2.2.2] octane). J. Am. Chem. Soc. 2017, 139, 10897-10902.

(45) Pan, Q.; Liu, Z.-B.; Tang, Y.-Y.; Li, P.-F.; Ma, R.-W.; Wei, R.-Y.; Zhang, Y.; You, Y.-M.; Ye, H.-Y.; Xiong, R. G. A Three-Dimensional Molecular Perovskite Ferroelectric: (3-Amoniopyrrolidinium) $\mathrm{RbBr}_{3} . \mathrm{J}$. Am. Chem. Soc. 2017, 139, 3954-3957.

(46) Ye, H.-Y.; Tang, Y.-Y.; Li, P.-F.; Liao, W.-Q.; Gao, J.-X.; Hua, X.N.; Cai, H.; Shi, P.-P.; You, Y.-M.; Xiong, R. G. Metal-Free ThreeDimensional Perovskite Ferroelectrics. Science 2018, 361, 151-155.

(47) Liu, S.; Sun, Z.; Ji, C.; Li, L.; Zhao, S.; Luo, J. Exceptional Bi-Step Switching of Quadratic Nonlinear Optical Properties in a OneDimensional Channel Compound. Chem. Commun. 2017, 53, 76697672 . 
(48) Yang, C.-S.; Tan, Y.-H.; Wang, C.-F.; Chen, S.-P.; Wang, B.; Wen, H.-R.; Tang, Y.-Z. Exceptional Second Harmonic Generation Responses, Switchable Dielectric Behaviours, and Ferroelectric Property in an Adduct of Hexamethylene-Tetramine-Bisnopinic acid. Chem. Phys. 2018, 502, 66-71.

(49) Molak, A.; Paluch, M.; Pawlus, S.; Klimontko, J.; Ujma, Z.; Gruszka, I. Electric Modulus Approach to the Analysis Electric Relaxation in Highly Conducting $\left(\mathrm{Na}_{0.75} \mathrm{Bi}_{0.25}\right)\left(\mathrm{Mn}_{0.25} \mathrm{Nb}_{0.75}\right) \mathrm{O}_{3}$ Ceramics. J. Phys. D: Appl. Phys. 2005, 38, 1450-1460.

(50) Xu, G.-C.; Ma, X.-M.; Zhang, L.; Wang, Z.-M.; Gao, S. DisorderOrder Ferroelectric Transition in the Metal Formate Framework of $\left[\mathrm{NH}_{4}\right]\left[\mathrm{Zn}(\mathrm{HCOO})_{3}\right]$. J. Am. Chem. Soc. 2010, 132, 9588-9590.

(51) Asadi, K.; Van der Veen, M. A. Ferroelectricity in Metal-Organic Frameworks; Characterization and Mechanisms. Eur. J. Inorg. Chem. 2016, 2016, 4332-4344.

(52) Boström, H. L. B.; Senn, M. S.; Goodwin, A. L. Recipies for Improper Ferroelectricity in Molecular Perovskites. Nat. Commun. 2018, 9, 2380.

(53) Scott, J. F. Ferroelectrics Go Bananas. J. Phys.: Condens. Matter 2008, 20, 021001.

(54) Sieradzki, A.; Maczka, M.; Simenas, M.; Zaręba, J. K.; Gagor, A.; Balciunas, S.; Kinka, M.; Ciupa, A.; Nyk, M.; Samulionis, V.; Banys, J.; Paluch, M.; Pawlus, S. On the Origin of Ferroelectric Structural Phases in Perovskite-Like Metal-Organic Formate. J. Mater. Chem. C 2018, 6, 9420-9429.

(55) Aizu, K. Possible Species of Ferromagnetic, Ferroelectric and Ferroelastic Crystals. Phys. Rev. B 1970, 2, 754-772.

(56) Kubelka, P.; Munk, F. Ein Beitrag Zur Optik Der Farbanstriche. Z. Techn. Phys. 1931, 12, 593-601.

(57) Hsu, H.-P.; Li, L.-C.; Shellaiah, M. S.; Sun, K. W. Structural, Photophysical, and Electronic Properties of $\mathrm{CH}_{3} \mathrm{NH}_{3} \mathrm{PbCl}_{3}$ Single Crystals. Sci. Rep. 2019, 9, 13311.

(58) Tao, S.; Schmidt, I.; Brocks, G.; Jiang, J.; Tranca, I.; Meerholz, K.; Olthof, S. Absolute Energy Level Positions in Tin- and Lead-based Halide Perovskites. Nat. Commun. 2019, 10, 2560.

(59) Jang, D. M.; Kim, D. H.; Park, K.; Park, J.; Lee, J. W.; Song, J. K. Ultrasound Synthesis of Lead Halide Perovskites Nanocrystals. J. Mater. Chem. C 2016, 4, 10625-10629.

(60) Hayashi, T.; Kobayashi, T.; Iwanaga, M.; Watanabe, M. Exciton Dynamics Related with Phase Transitions in $\mathrm{CsPbCl}_{3}$ Single Crystals. J. Lumin. 2001, 94-95, 255-259.

(61) Ji, C.; Wang, S.; Li, L.; Sun, Z.; Hong, M.; Luo, J. The First 2D Hybrid Perovskite Ferroelectric Showing Broadband White-Light Emission with High Color Rendering Index. Adv. Funct. Mater. 2019, 29, 1805038.

(62) Smith, M. S.; Connor, B. A.; Karunadasa, H. I. Tuning the Luminescence of Layered Halide Perovskites. Chem. Rev. 2019, 119, 3104-3139.

(63) Mao, L.; Wu, Y.; Stoumpos, C. C.; Traore, B.; Katan, C.; Even, J.; Wasielewski, M. R.; Kanatzidis, M. G. Tunable White-Light Emission in Single-Cation-Templated Three-Layered 2D Perovskites $\left(\mathrm{CH}_{3} \mathrm{CH}_{2} \mathrm{NH}_{2}\right)_{4} \mathrm{~Pb}_{3} \mathrm{Br}_{10-\mathrm{x}} \mathrm{Cl}_{\mathrm{x}}$. J. Am. Chem. Soc. 2017, 139, 1195611963. 